Journal for ImmunoTherapy of Cancer

\title{
Multiparametric MRI of early tumor response to immune checkpoint blockade in metastatic melanoma
}

Doreen Lau (D) ,, Mary A McLean (D) ,, ${ }^{1,2}$ Andrew N Priest (D) ,1,3 Andrew B Gill (1) , ${ }^{1,2}$ Francis Scott, ${ }^{1}$ Ilse Patterson, ${ }^{3}$ Bruno Carmo, ${ }^{3}$ Frank Riemer, ${ }^{1}$ Joshua D Kaggie, ${ }^{1}$ Amy Frary, ${ }^{1}$ Doreen Milne, ${ }^{4}$ Catherine Booth, ${ }^{4}$ Arthur Lewis, ${ }^{5}$ Michal Sulikowski, ${ }^{5}$ Lee Brown, ${ }^{5}$ Jean-Martin Lapointe (i) , ${ }^{5}$ Luigi Aloj, ${ }^{1,6}$ Martin J Graves, ${ }^{1,3}$ Kevin M Brindle, ${ }^{7}$ Pippa G Corrie (D) , ${ }^{4}$ Ferdia A Gallagher (D) 1,2

To cite: Lau D, McLean MA, Priest AN, et al. Multiparametric MRI of early tumor response to immune checkpoint blockade in metastatic melanoma. Journal for ImmunoTherapy of Cancer 2021;9:e003125. doi:10.1136/ jitc-2021-003125

- Additional supplemental material is published online only. To view, please visit the journal online (http://dx.doi.org/10. 1136/jitc-2021-003125).

$\mathrm{PGC}$ and $\mathrm{FAG}$ are joint senior authors.

Accepted 06 August 2021
Check for updates

(C) Author(s) (or their employer(s)) 2021. Re-use permitted under CC BY. Published by BMJ.

For numbered affiliations see end of article.

\section{Correspondence to}

Dr Doreen Lau;

doreen.lau@oncology.ox.ac.uk

Professor Ferdia A Gallagher; fag1000@cam.ac.uk

Dr Pippa G Corrie; pippa.corrie@addenbrookes. nhs.uk

\section{ABSTRACT}

Background Immune checkpoint inhibitors are now standard of care treatment for many cancers. Treatment failure in metastatic melanoma is often due to tumor heterogeneity, which is not easily captured by conventional CT or tumor biopsy. The aim of this prospective study was to investigate early microstructural and functional changes within melanoma metastases following immune checkpoint blockade using multiparametric MRI.

Methods Fifteen treatment-naïve metastatic melanoma patients (total 27 measurable target lesions) were imaged at baseline and following 3 and 12 weeks of treatment on immune checkpoint inhibitors using: $T_{2}$-weighted imaging, diffusion kurtosis imaging, and dynamic contrastenhanced MRI. Treatment timepoint changes in tumor cellularity, vascularity, and heterogeneity within individual metastases were evaluated and correlated to the clinical outcome in each patient based on Response Evaluation Criteria in Solid Tumors V.1.1 at 1 year.

Results Differential tumor growth kinetics in response to immune checkpoint blockade were measured in individual metastases within the same patient, demonstrating significant intertumoral heterogeneity in some patients. Early detection of tumor cell death or cell loss measured by a significant increase in the apparent diffusivity $\left(D_{\text {app }}\right)(p<0.05)$ was observed in both responding and pseudoprogressive lesions after 3 weeks of treatment. Tumor heterogeneity, as measured by apparent diffusional kurtosis $\left(\mathrm{K}_{\mathrm{app}}\right)$, was consistently higher in the pseudoprogressive and true progressive lesions, compared with the responding lesions throughout the first 12 weeks of treatment. These preceded tumor regression and significant tumor vascularity changes $\left(K^{\text {trans }}, v_{\mathrm{e}}\right.$, and $\left.v_{\mathrm{p}}\right)$ detected after 12 weeks of immunotherapy $(p<0.05)$. Conclusions Multiparametric MRI demonstrated potential for early detection of successful response to immune checkpoint inhibitors in metastatic melanoma.

\section{BACKGROUND}

Immune checkpoint inhibitors targeting the cytotoxic T-lymphocyte antigen-4 (CTLA-4), programmed cell death receptor-1 (PD-1) and programmed cell death receptor-1 ligand (PD-L1) are improving outcomes for increasing numbers of patients with solid cancers. ${ }^{1}$ These drugs are now the standard of care for treating many cancers including metastatic melanoma. ${ }^{2}$ International trials testing anti-PD-1 antibodies alone or in combination with anti-CTLA-4 antibodies in metastatic melanoma reported objective response of up to $58 \%$ and only a complete response of $11.5 \%$ at a median follow-up of 12.2-12.5 months. ${ }^{3}$ Although durable remissions are achieved in some patients, approximately half of treated patients do not respond, while all treated patients are at risk of immune-mediated toxicity that can be both life changing and life threatening. ${ }^{4}$ In clinical practice, standard CT and MRI imaging are used for evaluation of treatment response, usually undertaken at 12 weekly intervals. Assessment of response in the first few months can be difficult and can be confounded by possible pseudoprogression, characterized by the enlargement of target measurable metastases followed by subsequent regression over time. Biomarkers that could aid clinical decisions in the early stages of treatment are currently lacking. ${ }^{6}$

Biomarkers derived from whole blood sampling and tumor biopsy do not reflect the spatiotemporal dynamics of tumor immune response to checkpoint inhibition due to the marked interpatient, intermetastatic and intratumoral heterogeneity present in melanoma. ${ }^{78}$ Pseudoprogression seen in a small number of patients receiving immune checkpoint inhibitors is difficult to distinguish from true tumor progression using size measurements alone on conventional CT. ${ }^{910}$ Functional imaging techniques have the potential to longitudinally characterize individual tumor response to immunotherapy and could potentially be used in 
the future to provide an early and accurate prediction of treatment response.

Several approaches have been investigated to date for imaging response to immune checkpoint inhibition. Positron emission tomography (PET) with the glucose analog 2-deoxy-2- $\left[{ }^{18} \mathrm{~F}\right]$ fluoro-D-glucose $\left({ }^{18} \mathrm{~F}-\mathrm{FDG}\right)$ has shown promise for long-term successful response monitoring: a complete metabolic response (CMR) with ${ }^{18}$ F-FDG uptake 1 year after commencing treatment is associated with an excellent progression-free survival compared with those patients who do not show CMR. ${ }^{11}$ However, it is not known whether ${ }^{18}$ F-FDG PET can detect early response to treatment, as it can be particularly difficult to distinguish tumor metabolism from glucose uptake associated with immune infiltration after the initial introduction of immune checkpoint inhibitors. ${ }^{12}$ Although zirconium-89 radiolabeled antibodies targeting CD8, PD-1, and PD-L1 have been developed as tracers for first-in-human trials in experimental medicine studies, ${ }^{13-15}$ these radiolabeled approaches are expensive and cannot be easily implemented as routine clinical tools.

MRI is a widely available clinical imaging tool. The technique is particularly well suited for longitudinal tracking of early treatment response, as it does not involve exposure to ionizing radiation. ${ }^{16} 17$ Dynamic contrastenhanced MRI (DCE-MRI) measures properties of tissue vasculature $^{18}$ and is increasingly used in the diagnosis, staging, and treatment response assessment of many cancers. ${ }^{19}$ Pharmacokinetic modeling of the $\mathrm{T}_{1}$-weighted contrast-enhanced images provide quantitative measurements of tissue perfusion and vascular permeability (see online supplemental material for a detailed explanation of these parameters). For example, $K^{\text {trans }}$ is the volume transfer coefficient from the blood plasma space into the extravascular tumor interstitial space reflecting vascular permeability, which has been shown to change following successful treatment in a number of cancer types and therapeutic regimens..$^{21} \mathrm{v}_{\mathrm{e}}$ is the fractional volume of the extravascular-extracellular space, and $v_{p}$ is the vascular plasma volume. Following immunotherapy, DCE-MRI has been shown to detect tumor perfusion or vascular permeability as a surrogate biomarker of early tumor immune rejection in preclinical models of adoptive $\mathrm{T}$ cell therapy ${ }^{22} 23$ and has been foun to distinguish pseudoprogression from true tumor progression in patients with previously irradiated melanoma brain metastases after three cycles of ipilimumab. ${ }^{24}$

Diffusion-weighted imaging (DWI) is a complementary approach based on the molecular movement of water in tissues, which has been widely used for probing changes in cell density due to tumor cell death that occur following successful treatment in cancer. ${ }^{1725}$ An advanced DWI approach termed diffusion kurtosis imaging (DKI) has been shown to detect tumor cellularity and heterogeneity in many cancer types based on the non-Gaussian movement of water within the heterogeneous tumor microenvironment. ${ }^{26}{ }^{27}$ Cell density can be quantified on DKI based on the apparent diffusivity of water $\left(\mathrm{D}_{\text {app }}\right)$, and the microscopic heterogeneity of this water diffusion in tissue can be probed using a dimensionless metric termed apparent diffusion kurtosis $\left(\mathrm{K}_{\text {app }}\right)$. A more detailed explanation of these parameters can be found in online supplemental material. ${ }^{27} 28$

Here, we have used a multiparametric imaging approach combining morphological volumetric measurements with DCE and DKI to phenotype the microstructural and functional changes that occur in melanoma metastases before, during, and after treatment with immune checkpoint inhibitors.

In this prospective study, early changes in the growth kinetics, cellularity, heterogeneity and vascularity of the tumor microenvironment following immune checkpoint blockade between patients and between intermetastatic lesions were evaluated using multiparametric MRI (mpMRI). Metastatic melanoma offers a paradigm model to test the feasibility of these imaging methods in patients undergoing cancer immunotherapy.

\section{METHODS \\ Study design}

Patients were recruited for mpMRI as part of the MelResist study, which evaluated response and resistance biomarkers in metastatic melanoma patients undergoing systemic therapy. Written informed consent was obtained from all patients before enrolment. Patient eligibility criteria for undertaking MRI included: (A) clinical diagnosis of unresectable and previously untreated metastatic melanoma (American Joint Committee on Cancer Stage IV); (B) a treatment plan to commence standard immune checkpoint inhibitors as first-line therapy for unresectable metastatic melanoma; (C) Eastern Cooperative Oncology Group performance status score of 0 or 1 and life expectancy of 12 weeks or greater; (D) measurable disease on baseline CT (tumor diameter $>1 \mathrm{~cm}$ ); (E) availability of recent excised or biopsied tissue samples from metastatic tumors for histopathological confirmation; (F) known BRAF V600 mutation status; and $(G)$ no contraindication to undertaking MRI.

Enrolled patients received one of the following regimens: (A) anti-PD-1 monotherapy, $2 \mathrm{mg} / \mathrm{kg}$ or $200 \mathrm{mg}$ flat dose of pembrolizumab (Keytruda) every 3 weeks; or $3 \mathrm{mg} / \mathrm{kg}$ or $240 \mathrm{mg}$ of nivolumab (Opdivo) every 2 weeks or $480 \mathrm{mg}$ every 4 weeks and (B) combined antiCTLA-4 and anti-PD-1 therapy, $3 \mathrm{mg} / \mathrm{kg}$ of ipilimumab (Yervoy) plus $1 \mathrm{mg} / \mathrm{kg}$ of nivolumab (Opdivo) every 3 weeks for four cycles followed by nivolumab $240 \mathrm{mg}$ every 2 weeks or $480 \mathrm{mg}$ every 4 weeks. All treatments were administered by intravenous infusion. Treatment continued until disease progression (as defined by the 3 monthly restaging CT scans), development of unacceptable adverse side effects such as autoimmune disorders or patient withdrawal of consent. A schematic diagram for the mpMRI study flow chart and the clinical characteristics of the study participants are as shown in figure 1 and 
A

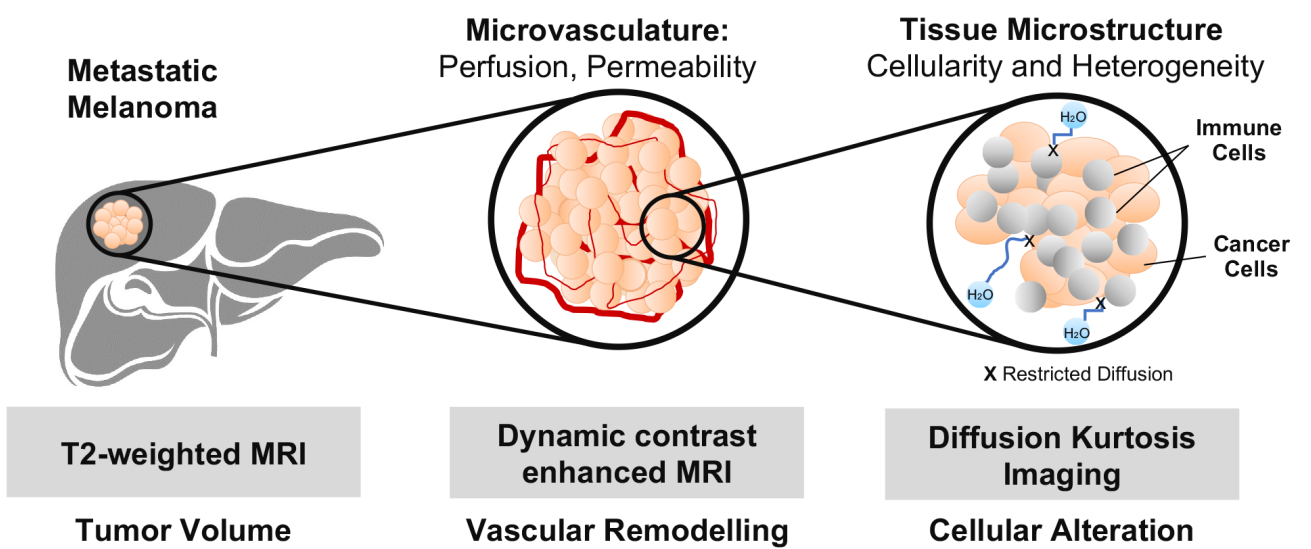

B

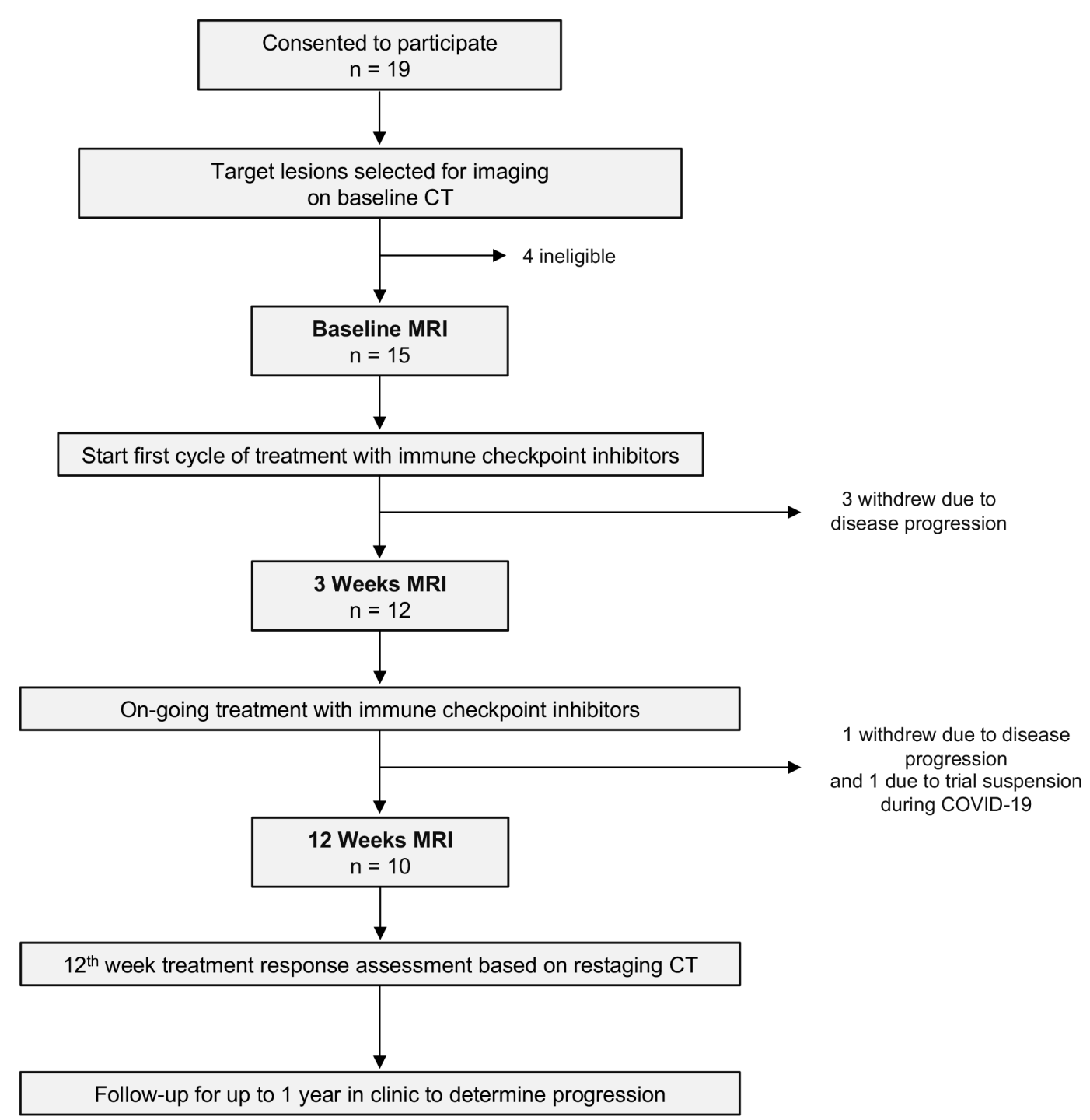

Figure 1 An mpMRI approach for longitudinal tracking of biological changes within tumors in response to immune checkpoint blockade. (A) Schematic diagram of the mpMRI approaches used in this study for monitoring tumor response to immune checkpoint blockade. $\mathrm{K}^{\text {trans }}$ measurements on dynamic contrast-enhanced MRI were used to quantify vascular permeability, while $v_{e}$ and $v_{p}$ reported on the volume of the extravascular-extracellular and vascular spaces, respectively. Diffusional kurtosis imaging, as an advanced form of diffusion-weighted imaging, was used to probe tissue microstructure using the metrics of apparent diffusivity $\left(D_{\text {app }}\right)$ as a measure of cellularity and apparent kurtosis $\left(K_{\text {app }}\right)$ for tissue heterogeneity. (B) Study flow chart for the melanoma immunotherapy trial (MelResist). mpMRI, multiparametric MRI. 
Table 1 Clinical characteristics of study participants

\begin{tabular}{|c|c|}
\hline \multicolumn{2}{|l|}{ Characteristics } \\
\hline No. of patients & 15 \\
\hline Age (median age, range) & $65.4(69,48-76)$ \\
\hline Gender & 10 males, 5 females \\
\hline AJCC Stage & IV \\
\hline \multicolumn{2}{|l|}{ ECOG performance status } \\
\hline 0 & 9 \\
\hline 1 & 6 \\
\hline \multicolumn{2}{|l|}{ BRAF status } \\
\hline BRAF V600 mutant & 3 \\
\hline BRAF Wild-type & 12 \\
\hline \multicolumn{2}{|l|}{ Serum LDH $(I U / m L)$ at baseline } \\
\hline Normal (<250) & 10 \\
\hline Elevated (>250) & 5 \\
\hline \multicolumn{2}{|l|}{ Neutrophils-to-lymphocyte ratio at baseline } \\
\hline Normal $(<5)$ & 12 \\
\hline High $(>5)$ & 3 \\
\hline \multicolumn{2}{|l|}{ Immunotherapy, n (\%) } \\
\hline Pembrolizumab & $6(40.0)$ \\
\hline Nivolumab & $2(13.3)$ \\
\hline Combined ipilimumab and nivolumab & $7(46.7)$ \\
\hline \multicolumn{2}{|c|}{ RECIST $1.1 \mathrm{CT}$ evaluation at 12 th week, $\mathrm{n}(\%)$} \\
\hline Partial response & $5(33.3)$ \\
\hline Stable disease & $4(26.7)$ \\
\hline Progressive disease & $6(40.0)$ \\
\hline \multicolumn{2}{|l|}{ RECIST $1.1 \mathrm{CT}$ evaluation at 1 year, $\mathrm{n}(\%)$} \\
\hline Complete response & $3(20.0)$ \\
\hline Partial response & $4(26.7)$ \\
\hline Progressive disease & $8(53.3)$ \\
\hline \multicolumn{2}{|l|}{ Anatomical site selected for MRI } \\
\hline Head and neck & 2 \\
\hline Chest & 1 \\
\hline Abdomen and pelvis & 7 \\
\hline Subcutaneous & 4 \\
\hline Limbs & 1 \\
\hline
\end{tabular}

All patients imaged were histologically confirmed as AJCC Stage IV melanoma.

AJCC, American Joint Committee on Cancer staging (seventh edition); ECOG, Eastern Cooperative Oncology Group; LDH, lactate dehydrogenase; RECIST

1.1, Response Evaluation Criteria in Solid Tumors guidelines V.1.1.

table 1. Further details on the patient demographics can be found in online supplemental table $\mathrm{S} 1$.

\section{Magnetic resonance imaging}

All patients underwent proton $\left({ }^{1} \mathrm{H}\right)$ MRI on a 3.0 Tesla system (Discovery MR750, GE Healthcare, Waukesha, Wisconsin, USA) using a 32-channel phased-array coil with respiratory gating or multiple breath-holds used to reduce motion artifacts during image acquisition for lesions in the abdomen. The mpMRI protocol included multiplanar $\mathrm{T}_{2}$-weighted single-shot fast spin-echo anatomical imaging, DKI of tumor cellularity and heterogeneity, and
DCE-MRI of tumor perfusion or vascular permeability. Imaging was conducted at three timepoints: within 1 week prior to starting treatment with immune checkpoint inhibitors (baseline MRI); 3 weeks after the first infusion (3-week MRI) and 12 weeks after the start of treatment (12-week MRI) coinciding approximately with the first standard-of-care restaging CT response assessment at 12 weeks. Further details on the imaging acquisition, image processing and analysis can be found in the online supplemental material and table $\mathrm{S} 2$.

\section{Classification of target melanoma metastases and measurement of response}

Conventional objective response of the target metastases was determined by measuring the best treatment outcome at the 12-week restaging CT and reassessed at 1 year if the patient survived. Response was evaluated by standard Response Evaluation Criteria in Solid Tumors (RECIST) V.1.1 guidelines at 12 weeks and 1 year. $^{29}$

In addition, tumor measurements were assessed using MRI during the first 12 weeks. Metastases with at least a $30 \%$ decrease in volume on the 12-week MRI were classified as responding, metastases with at least a $20 \%$ increase in volume were identified as true progression, while metastases with at least a $20 \%$ increase in volume at the 3-week MRI, but which subsequently decreased in $>30 \%$ on the 12-week scan, were classified as pseudoprogression.

\section{Statistical analysis}

Statistical analysis was performed in GraphPad Prism software V.8 (La Jolla, California, USA). All values were expressed as median and IQR to account for sample size differences between groups. Normality was assessed using the Shapiro-Wilk test. Changes in individual lesion mpMRI biomarkers over the treatment timepoints were evaluated using either paired t-test for normally distributed data or Wilcoxon matched-pair signed-rank test for data with non-parametric distribution. Differences between the subgroups of responding, pseudoprogressive, and true progressive lesions were evaluated using one-way analysis of variance for normally distributed data, or the Kruskal-Wallis test with Dunn's multiple comparison for non-parametric testing. Spearman's correlation analysis was used for evaluating any relationship between the mpMRI biomarkers across treatment timepoints. A value of $\mathrm{p}<0.05$ was considered as statistically significant.

\section{RESULTS}

\section{Clinical characteristics}

Fifteen treatment-naive patients (10 males, 5 females; median age 65 years) were imaged with mpMRI over the first 12 weeks of immunotherapy. Ten patients completed MRI at all three imaging timepoints (baseline, 3-week and 12-week MRIs); 5 patients were scanned at baseline and/ or 3 weeks before withdrawal from the trial due to clinical reasons such as early disease progression or clinical deterioration incompatible with continuing on the study. An 
additional four patients were enrolled on the study but were deemed as ineligible for the prospective trial due to insufficient time for scheduling of imaging scans before the start of treatment (within a week) or target lesions that were too small (less than $1 \mathrm{~cm}$ in largest diameter) for multiple timepoint imaging and follow-up treatment response assessment. 53\% of the patients received PD-1 monotherapy, while $47 \%$ of the patients were treated with combined CTLA-4 and PD-1 therapy.

\section{Differential response to immune checkpoint blockade}

Based on RECIST V.1.1 assessment at the 12-week restaging CT, five patients demonstrated partial response to immune checkpoint inhibitors, four had stable disease, and six showed disease progression. The patients with stable disease demonstrated differential response between the individual metastases. Two out of these four patients subsequently progressed at the 1-year restaging $\mathrm{CT}$, and the remaining two patients demonstrated continued response to treatment (table 1). Consequently, at the 1-year timepoint, three patients showed complete response, four demonstrated partial response, one was alive with progressive disease, and the remaining seven had died from progressive disease. Further details on patient demographics can be found in online supplemental figure 1 .

The mpMRI images for a total number of 27 enhancing target melanoma metastases that were first identified as more than $1 \mathrm{~cm}$ in diameter on staging CT were analyzed. In this study, a total of 13 responding, 4 pseudoprogressive, and 10 true progressive metastases were identified by MRI. Tumors were categorized into three subgroups (responding, pseudoprogression, and true progression) were based on comparing the 3-week MRI with the 12-week MRI, confirmed with restaging CT at 12 weeks and follow-up on the clinical outcome for up to 1 year. There were no lesions that showed a $30 \%$ decrease in volume at the 3-week MRI, which subsequently increased in volume at the 12-week MRI or on the restaging CT.

$\mathrm{T}_{2}$-MRI volumetric analysis showed differential interpatient and intermetastatic response to immune checkpoint blockade. Within the cohort of patients in our study, intermetastatic differences in the individual tumor growth kinetics were particularly evident in patients undergoing anti-PD-1 monotherapy, as compared with patients receiving combined CTLA-4 and PD-1 treatment, where response was almost immediate at the 3-week MRI (figure 2A), which may represent the fact that monotherapy takes longer to mount antitumor effects compared with combination therapy. Interestingly, increasing $T_{2}$ hyperintensity or inflammatory changes were detected within all four enlarged pseudoprogressive tumors at 3 weeks, which resolved at 12 weeks with a corresponding reduction in tumor volume (figure $2 \mathrm{~B}$ and figure 3 ).
Tumor cell death and changes in heterogeneity in response to treatment

Figure 4 and online supplemental figure S1 show the changes in tumor cellularity and heterogeneity measured on DKI. No significant difference in the average $\mathrm{D}_{\text {app }}$ for each patient, as a measure of tumor cell density, was detected between the responding and non-responding patients at baseline (figure $4 \mathrm{~A}$ ); median $\mathrm{D}_{\text {app }}$ of 1.44 for responding patients versus 1.33 for non-responding patients, $\mathrm{p}=0.62$ ). There was a significant increase in the average $\mathrm{D}_{\text {app }}$ of imaged target metastases for each patient representing reduced tumor cellularity $(p<0.05)$ in the responding patients at 3 weeks (median $\mathrm{D}_{\text {app }} 1.65$; IQR 1.59-1.77) compared with baseline (1.44; IQR 1.26-1.63), with a further significant increase at 12 weeks $(2.01$; IQR 1.60-2.22). In contrast, there was no significant change in $\mathrm{D}_{\text {app }}$ in the tumors of non-responders over the 12 weeks of treatment (figure 4B).

Further analysis based on classification of individual metastases from all patients into the three subgroups of 'responding', 'pseudoprogression' and 'true progression', showed a significantly lower $\mathrm{D}_{\text {app }}$, reflecting higher tumor cell density at baseline in the pseudoprogressive lesions (median 1.17; IQR 1.02-1.20), as compared with the responding (median 1.48; IQR 1.44-1.68; $\mathrm{p}<0.001$ ) and true progressive lesions (median 1.44; IQR 1.15-1.82; $\mathrm{p}<0.05$ ). Individual tumors responded differently to treatment: most of the responding and pseudoprogressive lesions exhibited a significant percentage increase in $\mathrm{D}_{\text {app }}$ at the 3-week MRI relative to baseline, indicating lower cellularity in most responding lesions (median increase in $\mathrm{D}_{\text {app }}$ by $8.9 \%$; IQR $2.3 \%-27.6 \%$; $\left.\mathrm{p}<0.05\right)$ and pseudoprogressive lesions (median increase by $48.0 \%$; IQR $45.2 \%-63.1 \% ; \mathrm{p}<0.05)$. A further increase in $\mathrm{D}_{\text {app }}$ was detected within the tumor microenvironment in most of the metastases responding at 12 weeks $(31.7 \%$; IQR $1.9 \%-45.5 \% ; \mathrm{p}<0.05)$. However, one lesion demonstrated higher cellularity (increase in $\mathrm{D}_{\text {app }}$ ) despite a reduction of tumor volume over 12 weeks of treatment; interestingly, this lesion subsequently increased in size at the sixth month restaging CT and was verified to be a pseudoprogressive lesion over a longer timeframe. Higher cellularity was also detected on average in the pseudoprogressive lesions at 12 weeks compared with the responding lesions, despite a reduction in tumor volume, which may reflect a later phase of immune infiltration and tumor cell killing in these metastases (figures 4 and 5). This patient demonstrated complete response on RECIST V.1.1 evaluation at the 1-year follow-up CT scan. Overall, the $\mathrm{D}_{\text {app }}$ values measured from the experimental DKI images correlated to the apparent diffusion coefficient (ADC) values obtained on clinical DWI for all imaged patients (online supplemental figure S2).

No significant difference in microscopic tumor heterogeneity at baseline $(\mathrm{p}=0.27)$ was detected in the tumors of the responders (median $\mathrm{K}_{\text {app }}$ 0.61; IQR 0.51-0.71), compared with non-responding patients (median 0.71; IQR $0.53-0.86$ ), as detected by the average apparent 
A

PD-1 Monotherapy

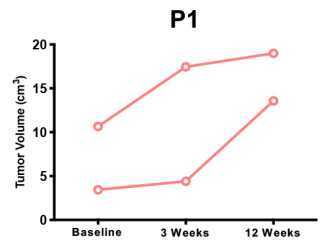

P2
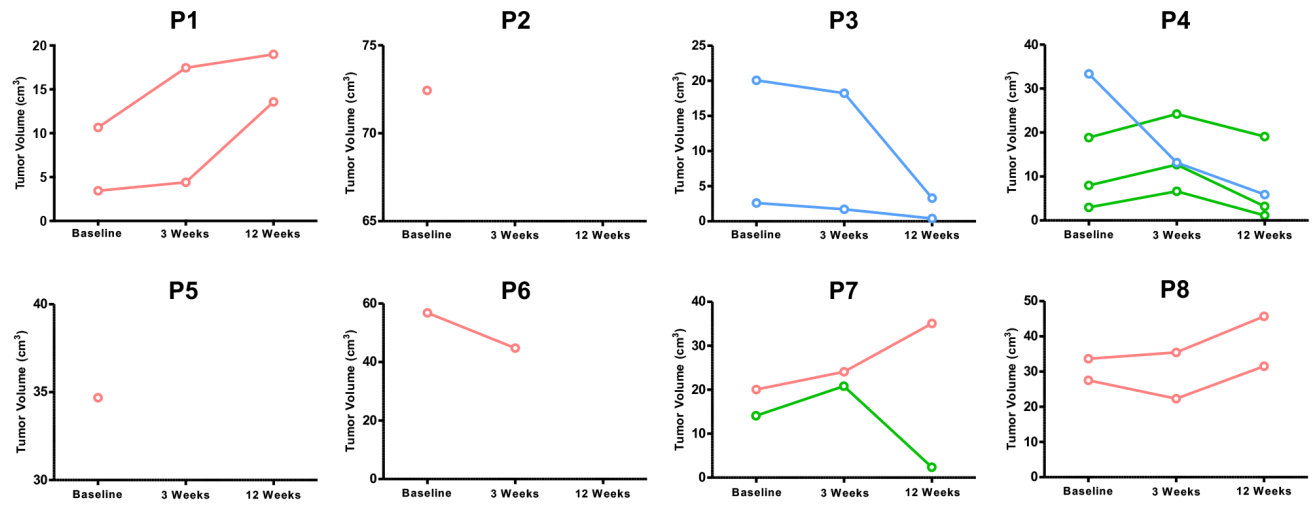

Combined CTLA-4 and PD-1 Therapy
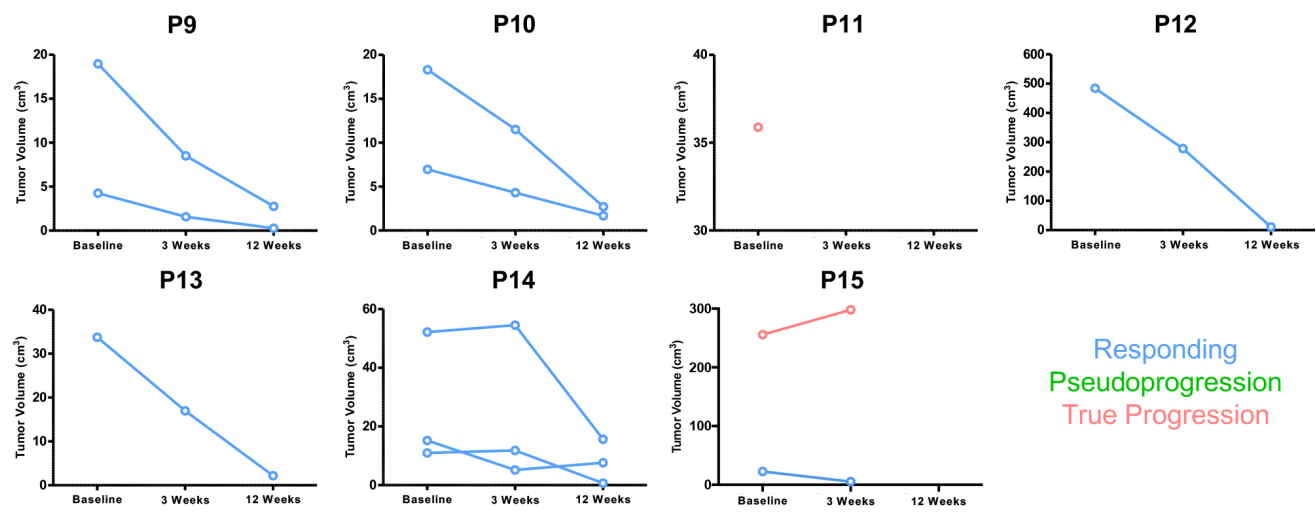

Responding

B
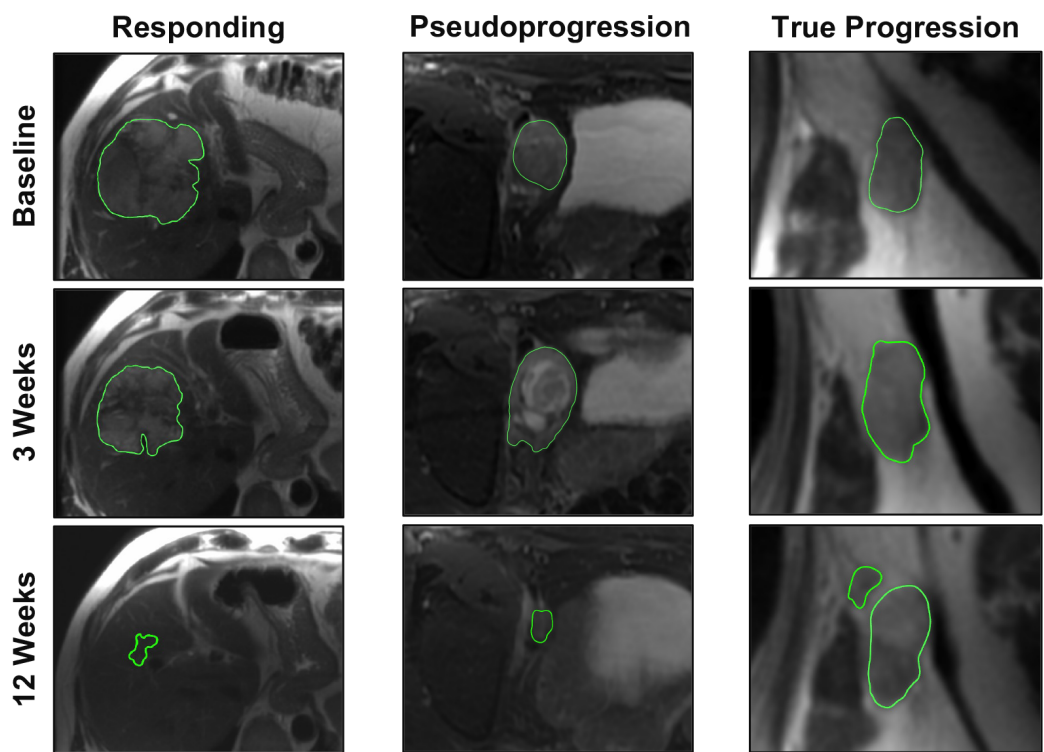

Figure 2 Interpatient and intermetastatic heterogeneity in response to immune checkpoint blockade. (A) Differential tumor growth kinetics in patients receiving PD-1 monotherapy compared with combined CTLA-4 and PD-1 treatment. Individual tumor volumes were measured on $\mathrm{T}_{2}$-weighted MRI. Categorization of tumors into three subgroups (responding, pseudoprogression, and true progression) were based on comparing the 3-week MRI with the 12-week MRI, confirmed with restaging CT at 12 weeks and follow-up on the clinical outcome for up to 1 year. (B) Representative $T_{2}$-weighted images from three patients with the classic features of responding, pseudoprogressive, and true progressive lesions. Note the T2 hyperintensity in keeping with inflammation in the pseudoprogressive lesion at 3 weeks. CTLA-4, cytotoxic T-lymphocyte antigen-4; PD-1, programmed cell death receptor- 1 . 
A

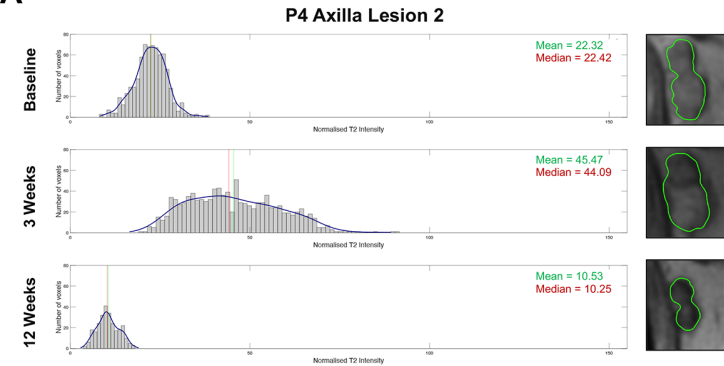

B

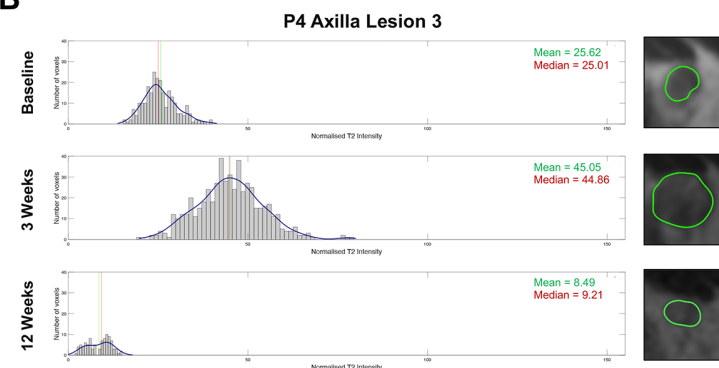

C

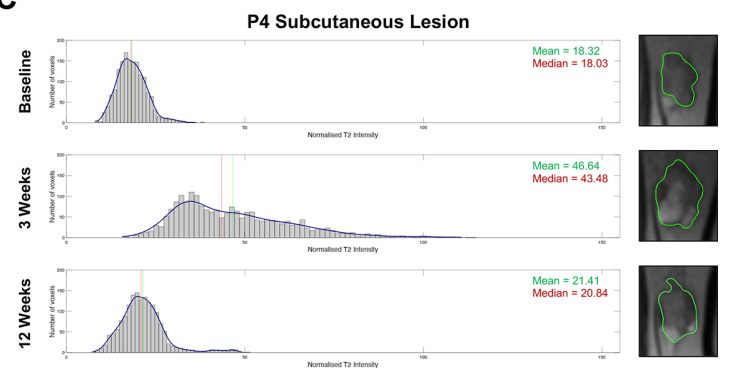

D

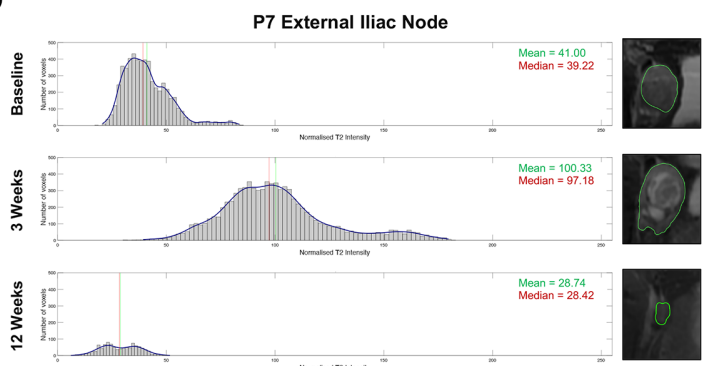

Figure 3 Histogram analysis of the $T_{2}$ intensity values of all four pseudoprogressive lesions. ${ }^{8}$ These included: the (A) axilla lesion 2, (B) axilla lesion 3, (C) subcutaneous lesion of patient 4, and the (D) external iliac node of patient 7.

kurtosis $\left(\mathrm{K}_{\mathrm{app}}\right)$ values from all target lesions for each patient obtained concurrently on DKI (online supplemental figure $\mathrm{S} 1)$. A significant reduction in $\mathrm{K}_{\text {app }}(\mathrm{p}<0.05)$ was detected in the tumors of responding patients at 3 weeks (median 0.59; IQR 0.51-0.65) compared with baseline (median 0.61; IQR 0.51-0.71). Further analysis of the individual lesions showed a trend towards a higher level of tumor heterogeneity, as measured by $\mathrm{K}_{\text {app}}$, in the pseudoprogressive lesions throughout the first 12 weeks of treatment (baseline: 0.84; 3 weeks: 0.64; 12 weeks: 0.76) compared with the responding lesions (baseline: 0.59; 3 weeks: 0.54 ; 12 weeks: 0.56 ); although this did not reach statistical significance, it may reflect underlying immune cell infiltration or cell death over the course of treatment. As with the results for $\mathrm{D}_{\text {app}}$, no significant change in $\mathrm{K}_{\text {app }}$ was detected in the progressing metastases during the first 12 weeks of treatment.

\section{Tumor vascular remodeling following cell death}

Figure 6 and online supplemental figure S3 show the changes in tumor vascularity and perfusion during 12 weeks of treatment, as measured by DCE-MRI and contrast kinetic modeling using the extended Tofts model. The average tumor vascular transfer constant $\left(\mathrm{K}^{\text {trans }}\right)$ at baseline was higher in the target lesions of the responding patients (median $\mathrm{K}^{\text {trans }} 0.56$; IQR 0.23-1.37) compared with the non-responders (0.15; IQR 0.11-0.44; $\mathrm{p}<0.05)$. Similarly, the average fractional extravascular-extracellular volume $\left(\mathrm{v}_{\mathrm{e}}\right)$ at baseline of the target lesions of the responding patients were higher (median $\mathrm{v}_{\mathrm{e}} 0.49$; IQR $0.31-0.77)$ compared with the non-responders (0.19; IQR $0.15-0.32 ; \mathrm{p}<0.05)$. A significant reduction in these tumor vascularity metrics $\left(\mathrm{K}^{\text {trans }}, \mathrm{v}_{\mathrm{e}}\right.$, and $\mathrm{v}_{\mathrm{p}}$ ) was only detected at 12 weeks compared with baseline $(0.11$; IQR $0.05-0.45$; $\mathrm{p}<0.05)$ but not at 3 weeks. A gradual increase in tumor vascular metrics was detected in the tumors of nonresponding patients over the course of treatment, but this was not statistically significant, which may reflect the small numbers, particularly at the 12-week MRI. Further analysis on the individual lesions showed no significant difference in the vascular transfer constant $\mathrm{K}^{\text {trans }}$, fractional volume of the extravascular-extracellular space $\mathrm{v}_{\mathrm{e}}$, or fractional plasma volume $\mathrm{v}_{\mathrm{p}}$, between the three subgroups of lesions before the start of treatment. A significant decrease in $\mathrm{K}^{\text {trans }}$ relative to baseline was detected in most responding lesions at 12 weeks (median $-66.19 \%$; IQR -92.00 to $-46.49 \%$; $\mathrm{p}<0.01)$ but not at the 3-week MRI $(-29.73 \%$; $\mathrm{IQR}-40.51$ to $14.01 \% ; \mathrm{p}=0.23)$. Similarly, $\mathrm{v}_{\mathrm{e}}$ and $\mathrm{v}_{\mathrm{p}}$ were also lower in the responding lesions at 12 weeks $(\mathrm{p}=0.07$ and $\mathrm{p}<0.01$, respectively). A trend towards lower $\mathrm{K}^{\text {trans }}$ was also detected in most pseudoprogressive lesions at 3 weeks (median 0.47; IQR 0.18-0.60) and 12 weeks $(0.15$; IQR 0.11-0.32), compared with baseline (0.52; IQR $0.19-0.82$ ), but this was not statistically significant given the small number of pseudoprogressive lesions within the patient cohort.

\section{Early treatment timepoint changes in tumor cellularity is independent of tumor volume}

Spearman's correlation analysis of the mpMRI biomarkers over the first 12 weeks of treatment in this cohort showed no significant correlation between tumor volume and $\mathrm{D}_{\text {app }}$ (online supplemental figure $\mathrm{S} 4$ ). This implies that the early detection of changes in tumor cellularity from immune cytotoxic killing of tumor cells at the 3-week MRI was independent of changes in tumor volume. However, a positive correlation was found between tumor volume and all metrics of tumor vascularity and perfusion at 12 weeks $\left(\mathrm{K}^{\text {trans }}, \mathrm{v}_{\mathrm{e}}\right.$, and $\left.\mathrm{v}_{\mathrm{p}}\right)$, suggesting that vascular remodeling 
A

Per Patient at Baseline

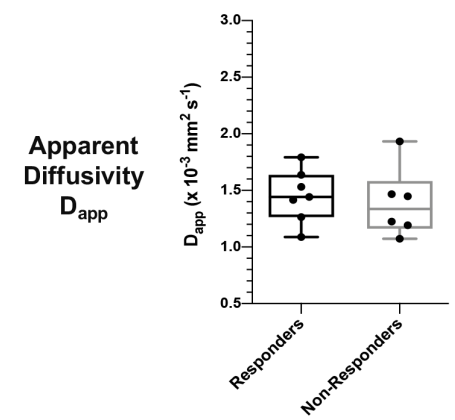

B

Responders

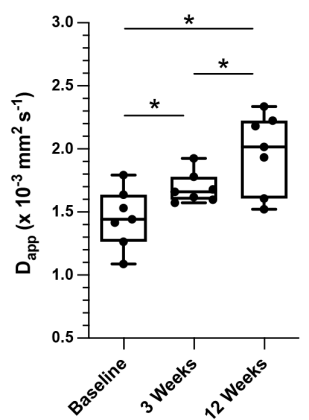

Non-Responders

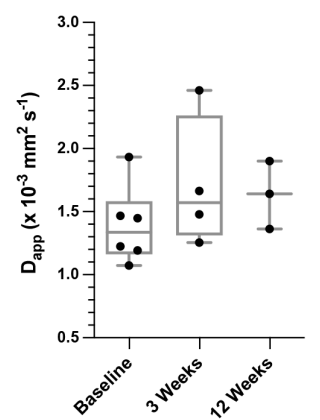

C

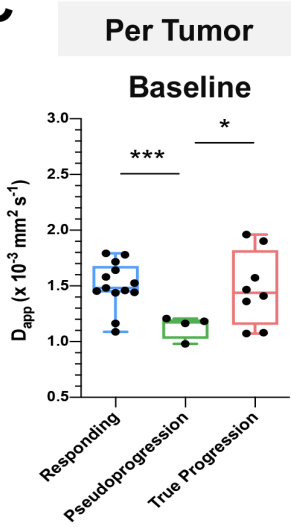

3 Weeks

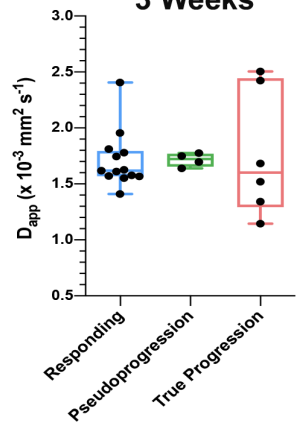

D

Responding

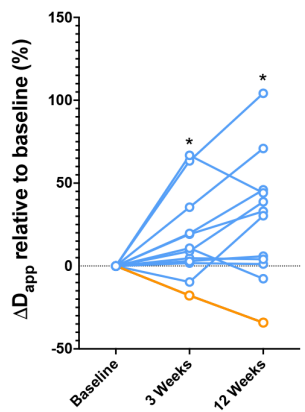

E

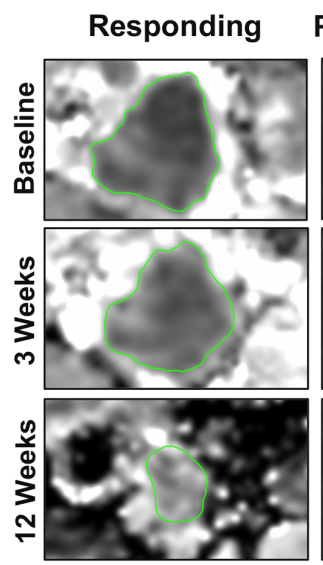

Pseudoprogression
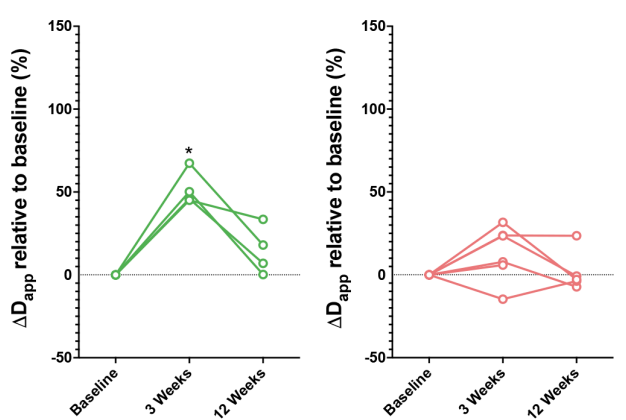

12 Weeks
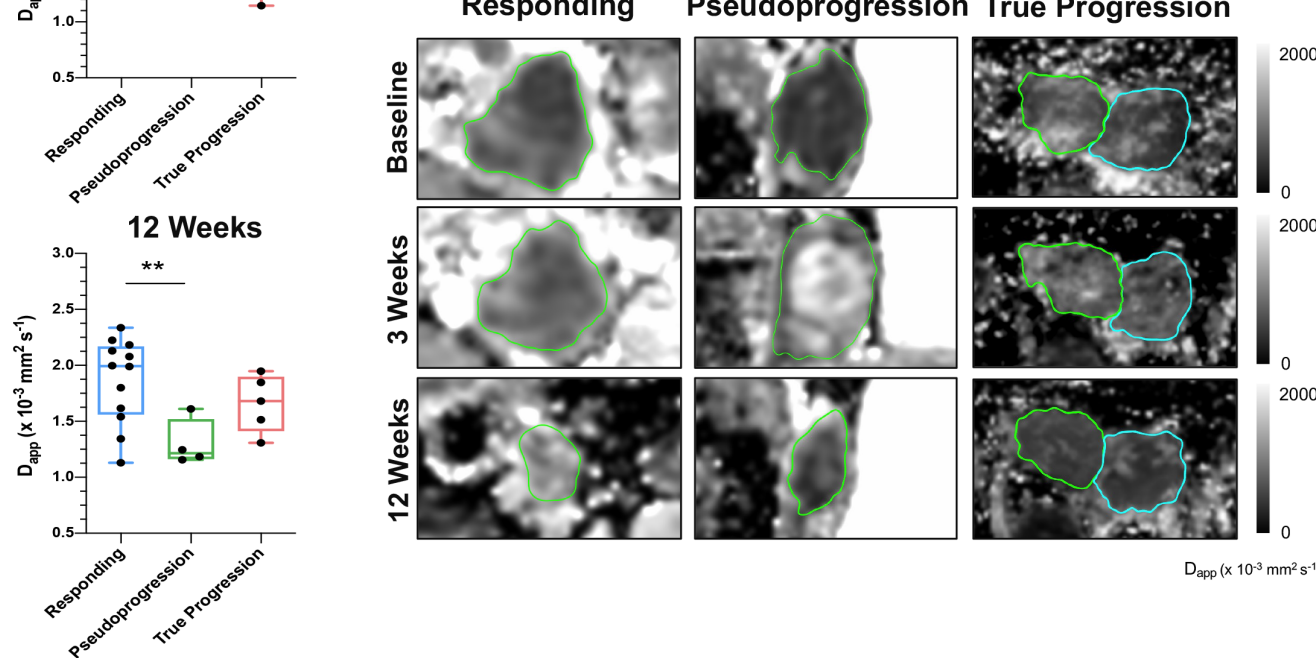

$D_{\text {app }}\left(\times 10^{-3} \mathrm{~mm}^{2} \mathrm{~s}^{-1}\right)$

Figure 4 Early detection of tumor cell death using DKI. (A) Comparison of apparent diffusivity $\left(D_{\text {apo }}\right)$ as a measure of tumor cell density between responders and non-responders at baseline before the start of treatment. (B) Changes in average tumor $\mathrm{D}_{\text {app }}$ on a per patient basis over the course of treatment, divided according to overall response. (C) Response of individual lesions classified into three subgroups (responding, pseudoprogression, and true progression) showing the differences in tumor cellularity at baseline. (D) Percentage change in $D_{\text {app }}$ relative to baseline in individual lesions from the three subgroups. (E) Representative $D_{\text {app }}$ images from three lesions categorized as responding, pseudoprogression, and true progression, respectively, based on the 1-year restaging CT. Data are presented as median and IQR. Normality was assessed using the Shapiro-Wilk test. Mann-Whitney test was performed to assess differences between two independent lesion subgroups; Kruskal-Wallis test with post hoc Dunn's multiple comparison analysis was performed to test for differences between three independent lesion subgroups; ${ }^{*} p<0.05$; ${ }^{* * *} p<0.001$. Yellow line in figure part $D$ indicates the percentage change in $D_{\text {app }}$ for patient 4. Analysis of apparent kurtosis as a measure of tumor heterogeneity, detected concurrently using DKI, is found in online supplemental figure S1. DKI, diffusion kurtosis imaging. 
A
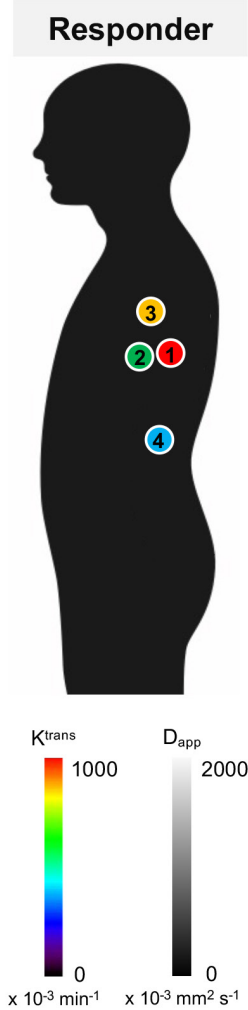

Baseline
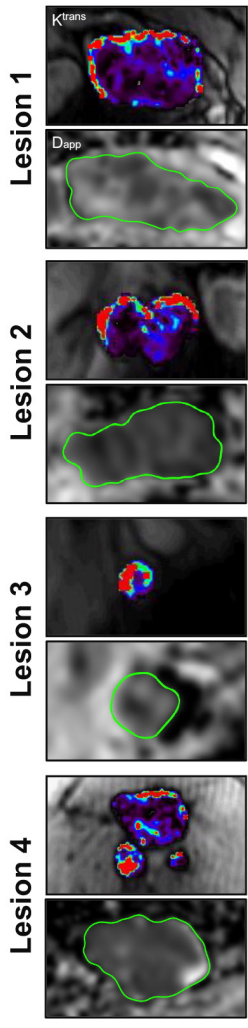

B

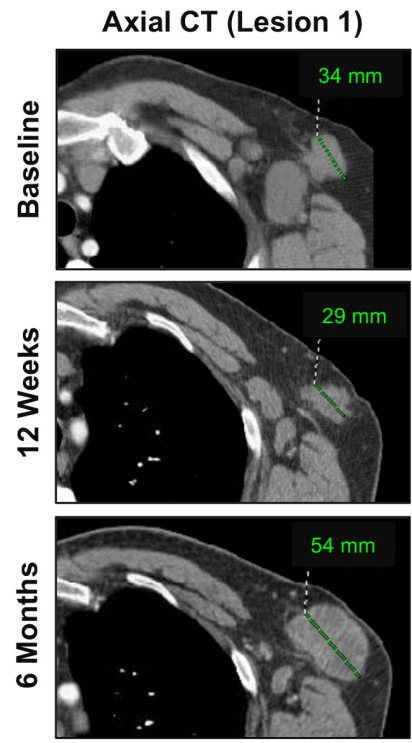

3 Weeks
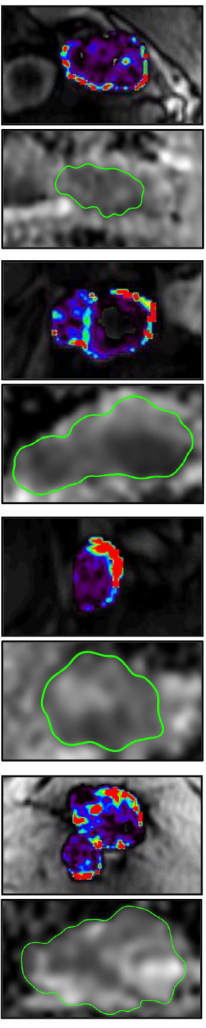

C
12 Weeks
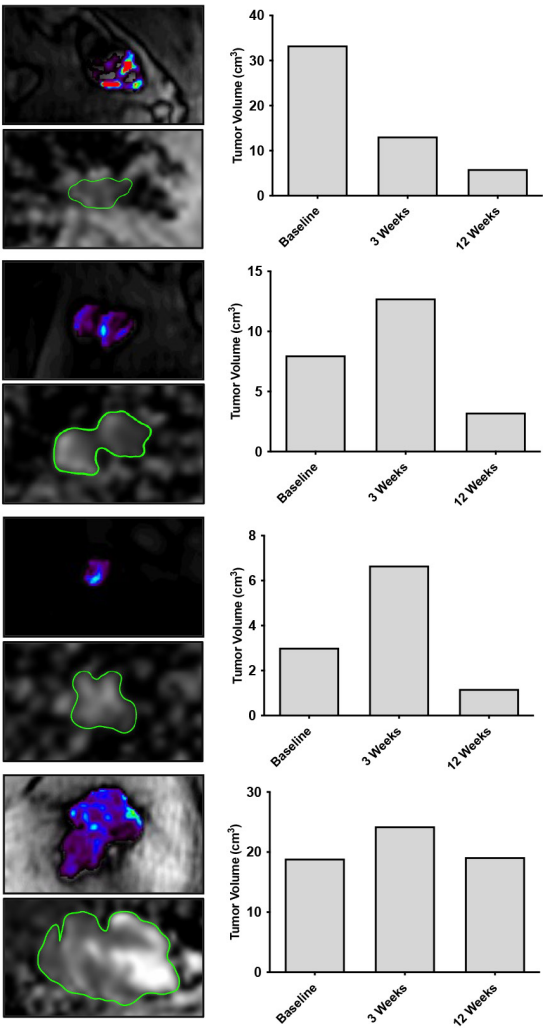

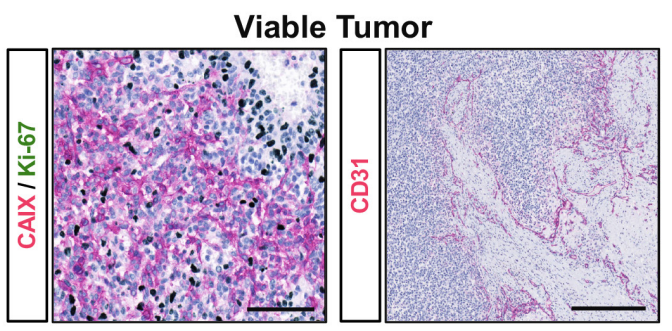

Largest diameter on CT

Figure 5 Dynamic changes in intertumoral response to immune checkpoint blockade within a single patient. (A) Multiple metastases in a patient on treatment with nivolumab (PD-1 monotherapy). Intertumoral differences in treatment response, vascular permeability, and cellularity were measured in four target lesions on MRI during the first 12 weeks of treatment. Increased cellularity was detected in the responding tumor (lesion 1) despite a reduction in tumor volume and lower vascularity at 12 weeks. The responding lesion subsequently progressed at 6 months on treatment and was surgically resected. (B) Axial CT of lesion 1 at baseline, 12 weeks, and 6 months; largest tumor diameter shown in $\mathrm{mm}$. (C) Immunohistochemistry of lesion 1 showed remarkable infiltration of immune cells (CD8) in viable tumor tissues that were highly hypoxic (CAIX), proliferative (Ki67), and vascular (CD31). Scale bars for CD8 immunostained images represent $100 \mu \mathrm{m}$ (10x magnification) and $50 \mu \mathrm{m}(80 \times$ magnification); $100 \mu \mathrm{m}$ in CAIX/Ki67 dual staining (20x magnification) and CD31 (10× magnification). PD-1, programmed cell death receptor-1. 

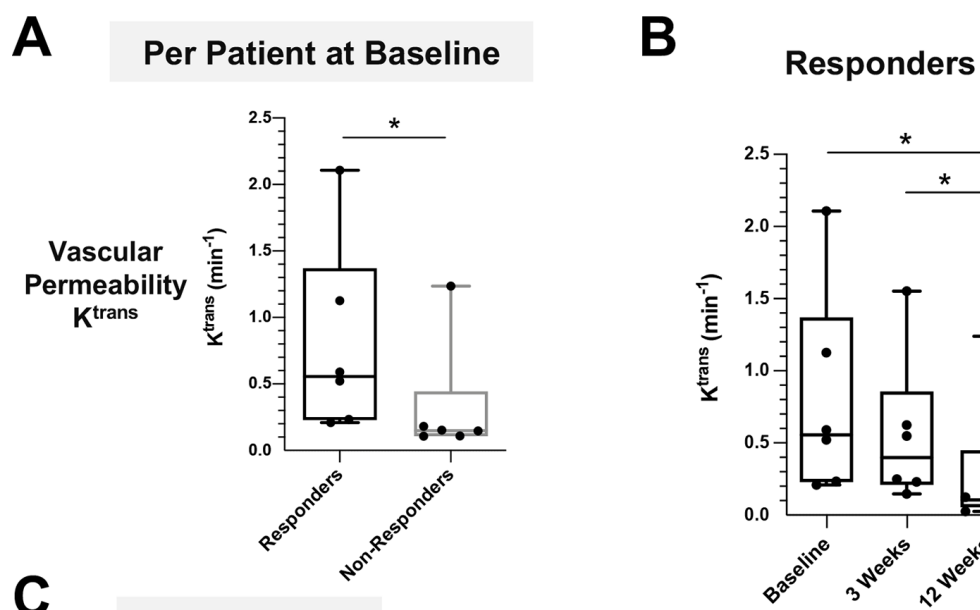

\section{Non-Responders}
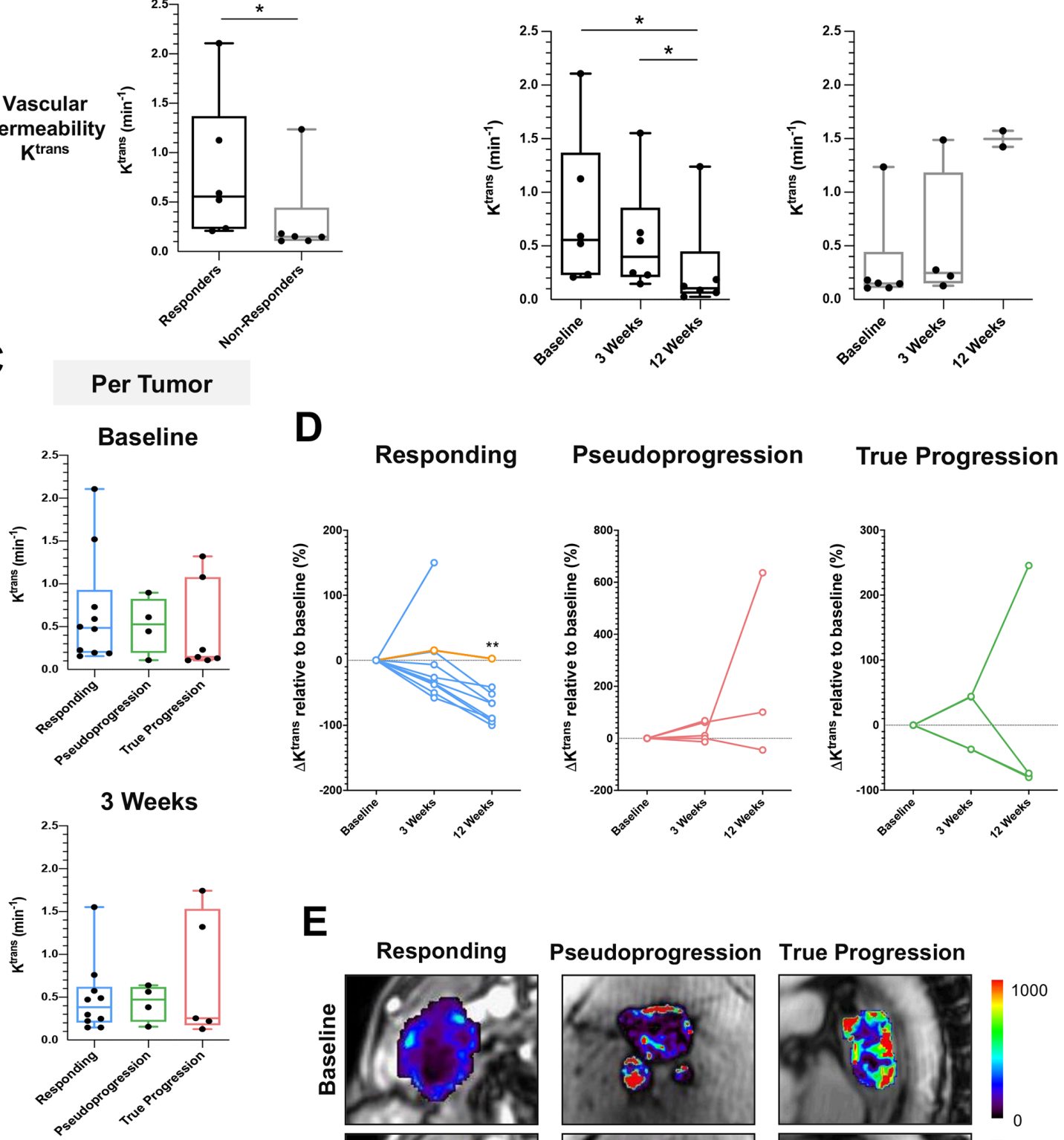

E
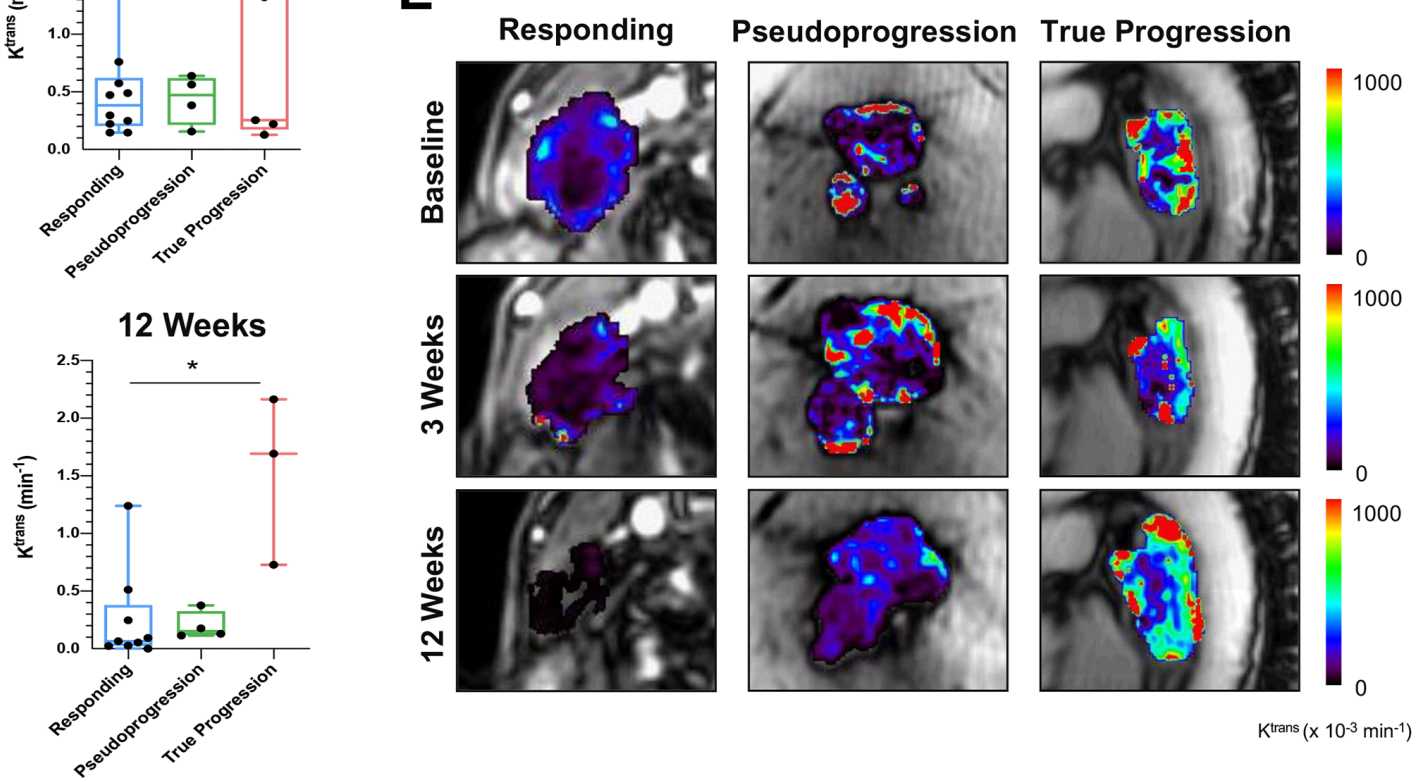

$K^{\text {trans }}\left(\times 10^{-3} \mathrm{~min}^{-1}\right)$

Figure 6 Tumor vasculature remodeling following immune cytotoxic killing and tumor cell death. (A) Comparison of vascular permeability $\mathrm{K}^{\text {trans }}$ between responders and non-responders at baseline before the start of treatment. (B) Changes in tumor $\mathrm{K}^{\text {trans }}$ over the course of treatment. (C) Comparison of tumor vascular permeability at baseline between individual lesions from the three subgroups: responding, pseudoprogression, and true progression. (D) Percentage change in $\mathrm{K}^{\text {trans }}$ relative to baseline in individual lesions from the three subgroups. (E) Representative $K^{\text {trans }}$ images from the three subgroups lesions. ${ }^{*} \mathrm{P}<0.05$; ${ }^{\star \star} \mathrm{P}<0.01$. Yellow line in figure part $\mathrm{D}$ indicates the percentage change in $\mathrm{K}^{\text {trans }}$ for patient 4 . Analysis for other $\mathrm{DCE}-\mathrm{MRI}$ parametric measurements is found in online supplemental figure S3. DCE-MRI, dynamic contrast-enhanced MRI. 
may be related to tumor size changes following immune checkpoint blockade.

\section{DISCUSSION}

As immune checkpoint inhibitors become more widely used in routine clinical practice, there is an unmet need for more effective tools to measure successful response to these agents. This is increasingly important as more patients with cancer are being offered long term immunotherapy, which is costly to healthcare systems and comes with a significant risk of side effects. Tumor heterogeneity is one of the major challenges for effective cancer treatment and manifest as morphological, functional, cellular, metabolic, and molecular diversity. ${ }^{30} 31$ Clinical tools to image this multilayered tumor heterogeneity and how it changes with immunotherapy could have a role in differentiating tumor resistance from successful response early in the treatment pathway. ${ }^{32}$

In this study, longitudinal tracking of microstructural and functional changes in metastatic melanoma during the first 12 weeks of treatment was performed using mpMRI. Heterogeneity in response to immune checkpoint blockade was observed in these treatment-naive tumors. This heterogeneity may be due to interpatient and intermetastatic differences in tumor immunogenicity, as some patients may have more delayed response, multiple waves of immune activation or ongoing immune evasion and clonal expansion during continuous treatment, as exemplified by patient 4 . These changes in the tumor microenvironment may not be identified by standard CT or MRI, as the change in tumor size alone are often insufficient to determine treatment benefit at the early stages of immunotherapy. Therefore, a clinically applicable tool to evaluate immunotherapy is required to guide clinical decision making. ${ }^{33}$

Treatment response to immune checkpoint blockade was captured longitudinally on mpMRI in this study using three approaches to assess the tumor microenvironment: $\mathrm{T}_{2}$-weighted MRI of tissue structure, DKI of cellular density and its microscopic heterogeneity, and DCE-MRI of the tumor vasculature. An interesting and unexpected observation was an increase in median $\mathrm{T}_{2}$-weighted signal intensity following 3 weeks of immunotherapy (one infusion of immunotherapy) in the pseudoprogressive metastases, compared with metastases that responded or were shown to progress at later timepoints. This is likely to be due to tumor enlargement from significant immune cell infiltration and inflammation, rather than tumor proliferation. $T_{2}$-weighted MRI represents a very simple routine clinical tool that may be able to discriminate pseudoprogression from true progression if this initial observation could be confirmed in larger studies. Quantitative analysis of these signal intensity changes, for example, using $\mathrm{T}_{2}$ mapping, may be useful in future trials for more detailed characterization of these microstructural changes. ${ }^{34}$

Changes in cell density were measured using $\mathrm{D}_{\text {app }}$ on DKI to detect either cytotoxic T-cell killing or increased cell density from immune infiltration or tumor proliferation. Cell loss measured as an increase in the median $\mathrm{D}_{\text {app }}$ was detected in both responding and pseudoprogressive lesions as early as 3 weeks after the start of treatment compared with baseline. Further reductions in cell density within the responding lesions were detected at the 12-week MRI. An increase in ADC measured on DWI (equivalent to the $\mathrm{D}_{\text {app }}$ measured here) has also been reported in previously treated ocular melanoma responding to immunostimulatory adenoviral CD40L gene therapy: $\geq 1$ fold change in ADC at week 5 following treatment was a better predictor of objective survival than metabolic changes on ${ }^{18}$ F-FDG PET and tumor size changes on MRI. ${ }^{35}$ Interestingly, in our study, an increase in cell density (or lower $\mathrm{D}_{\text {app }}$ ) was detected in the pseudoprogressive lesions at 12 weeks compared with the responding lesions, despite reduced tumor volumes measured on the 12-week MRI and standard restaging CT. No significant correlation between $\mathrm{D}_{\text {app }}$ and tumor volume was detected across the imaging timepoints, which implies that the estimation of cell density based on water diffusion within the tumor microenvironment is independent of tumor volume and is therefore an important additional metric to measure. A higher degree of tumor heterogeneity, as assessed by an increase in $\mathrm{K}_{\mathrm{app}}$, was detected in the pseudoprogressive lesions throughout the MRI imaging timepoints, compared with the responding lesions. This supports the hypothesis that there is underlying cellular alteration with different phases of immune activation and proliferation in the pseudoprogressive lesions over the course of treatment. Although there was a higher $\mathrm{K}_{\text {app }}$ in the true progressive lesions at all imaging timepoints compared with the responding lesions, the feasibility of using DKI alone to differentiate true progression from pseudoprogression could not be established as the number of nonresponders with complete MRI scans are limited in the metastatic disease setting due to early disease progression and withdrawal from the trial. Nevertheless, greater tumor heterogeneity at baseline (entropy, dissimilarity, and contrast texture features) measured on CT radiomics has been previously reported in non-responders to PD-1 monotherapy. ${ }^{36}$

The tumor vasculature plays a significant role in regulating tumor homeostasis, metastasis, and immune trafficking. ${ }^{37}$ The vascular networks in malignant tumors are typically disorganized with immature, tortuous, and leaky blood vessels that are hyperpermeable to intravascular contrast agents. DCE-MRI showed a gradual decrease in: the tumor vascular permeability $\mathrm{K}^{\text {trans }}$; the extravascularextracellular space $\mathrm{v}_{\mathrm{e}}$; and the plasma volume fraction $\mathrm{v}_{\mathrm{p}}$ within the responding lesions. This was more prominent at the 12-week MRI when a reduction in tumor burden was detected, suggesting that tumor vasculature remodeling and shutdown may have occurred following cell death caused by cytotoxic $\mathrm{T}$ cell killing. This contrasts with the effects of antiangiogenic treatments in human melanoma xenografts whereby the treatments are more directed towards the vascular network and are generally 
not cytotoxic. Tumor vasculature remodeling as represented by a lower $\mathrm{K}^{\text {trans }}$, often precedes cell death and reduction in tumor burden, with no significant change in cell density measurements such as $\mathrm{v}_{\mathrm{e}}$ or ADC. ${ }^{38}$ Despite the small number of pseudoprogressive lesions available for analysis in our study, lower $\mathrm{K}^{\text {trans }}, \mathrm{v}_{\mathrm{e}}$ and $\mathrm{v}_{\mathrm{p}}$, with a corresponding reduction in tumor volume because of cell death, was detectable in most pseudoprogressive lesions at 12 weeks. The pseudoprogressive lesions in general demonstrated lower vascular permeability and perfusion compared with the true progressive lesions at 12 weeks. Our findings are in concordance with a previous study assessing DCE-MRI melanoma brain metastases study in which lower $\mathrm{v}_{\mathrm{p}}$ was detected in previously irradiated pseudoprogressive lesions compared with true progressive lesions after three cycles of ipilimumab. ${ }^{24}$ This suggested that DCE-MRI may have utility in distinguishing true progressive lesions from treatment-responsive lesions but at a later timepoint compared with diffusion measurements. Interestingly, higher $\mathrm{K}^{\text {trans }}$ and $\mathrm{v}_{\mathrm{e}}$ were detected at baseline in all imaged tumors of most patients who were responders to immune checkpoint inhibitors compared with the non-responders. One explanation could be that the differences in tumor vasculature between tumors may play a role in determining immune trafficking and subsequent immune eradication of tumor cells. ${ }^{37}$ Ideally, this could be explored by tissue sampling of multiple lesions both before and during therapy, but this is not practical in the metastatic disease setting clinically, and further preclinical research is required.

As part of this prospective study, we have demonstrated marked intralesional, intermetastatic, and interpatient heterogeneity in melanoma over the first 12 weeks of immunotherapy. After only 3 weeks of treatment or one infusion of immunotherapy, a decrease in cellularity, as measured on DKI, could distinguish responding patients from non-responders, as well as individual responding and pseudoprogressing tumors from true progressing ones. An interesting finding was an increase in normalized $\mathrm{T}_{2}$-weighted signal and its distribution in the pseudoprogressing lesions compared with the progressing lesions after 3 weeks treatment. Therefore, combining conventional $\mathrm{T}_{2}$-weighted and DKI at 3 weeks after starting immunotherapy could be used to identify pseudoprogression during the early stages of treatment. Although there was higher tumor vascular permeability and perfusion at baseline in the responding patients on DCE-MRI compared with non-responders, changes in $\mathrm{K}^{\text {trans }}$ could not be used to distinguish responding and pseudoprogressing lesions until after 12 weeks of treatment showing that measurable vascular changes occur later than changes in cellularity.

Our study presented several strengths and limitations. This is the first prospective MRI study to serially track cellular and functional changes in melanoma metastases during immune checkpoint blockade. As all melanoma metastases analyzed in this study were previously untreated and unresectable tumors, the treatment timepoint changes measured on mpMRI were directly related to immunotherapeutic effects on individual lesions. Partial volume effects on image measurements were minimal as several patients in our trial presented large metastases at baseline. A stringent criterion for imaging and analysis was maintained to include only patients with measurable disease so that the biological changes were trackable over 12 weeks of immunotherapy. Nevertheless, our study is limited by the small sample size, which restricts the scope for wider interpretation of the results and evaluation of the imaging biomarkers for their predictive values. Future multicenter trials are required to test and validate these imaging biomarkers in a larger patient cohort, with the aim of integrating these imaging methods into immunotherapy trials and routine clinical management. Our study is further limited by the lack of radiological-pathological correlation, as relatively few metastases are readily accessible to biopsy. This difficulty in obtaining tumor tissues from metastatic sites further highlights the strengths of non-invasive imaging as a surrogate for pathology as changes in tumor growth kinetics, cell density, heterogeneity, and vascularity within individual tumors could be longitudinally tracked over the course of treatment.

Although no CT was available at the 3-week MRI timepoint for direct comparison, the lesional volume on MRI at this early timepoint represents a surrogate for the CT size measurements. Using size criteria alone, conventional CT is unlikely to provide additional information over MRI. The latter not only provides enhanced soft tissue contrast, but importantly can probe quantitative measures of tissue function that are not possible with CT, thus providing significant biological information as we have demonstrated here. Although CT can be used to probe tumor perfusion as part of dynamic contrastenhanced CT, this is usually at lower temporal resolution and with a significant radiation burden given the multiple acquisition timepoints. More generally, the radiation dose from CT decreases its suitability for multiple timepoint imaging, especially in patients who are particularly at risk of radiation effects. The emerging field of radiomics to study tissue heterogeneity would also be interesting to evaluate on CT at this early 3-week timepoint, but both radiomics and CT perfusion are currently research tools and require validation within future prospective trials.

In conclusion, mpMRI has shown potential for early assessment of response to immunotherapy in metastatic melanoma patients. Early changes in tumor cellularity measured on DKI following 3 weeks after starting treatment could be used to detect responding and pseudoprogressive melanoma metastases before a change in tumor volume and vascular permeability. This work could have important implications for monitoring treatment of metastatic melanoma and the increasing number of solid cancers treated with immunotherapy.

\section{Author affiliations}

${ }^{1}$ Department of Radiology, University of Cambridge, Cambridge, UK

${ }^{2}$ Cancer Research UK Cambridge Centre, Cambridge, UK 
${ }^{3}$ Department of Radiology, Addenbrooke's Hospital, Cambridge, UK

${ }^{4}$ Department of Oncology, Cambridge University Hospitals NHS Foundation Trust, Addenbrooke's Hospital, Cambridge, UK

${ }^{5}$ Clinical Pharmacology \& Safety Sciences, AstraZeneca PLC, Cambridge, Cambridgeshire, UK

${ }^{6}$ Department of Nuclear Medicine, Addenbrooke's Hospital, Cambridge, UK ${ }^{7}$ Cancer Research UK Cambridge Research Institute, Cambridge, UK

\section{Twitter Doreen Lau @DoreenLau4 and Ferdia A Gallagher @FerdiaGallagher}

Contributors Conceptualization of study: DL, FAG, PGC, MAM, MJG, and KMB. Data curation and formal analysis: DL, FAG, PGC, MAM, ANP, ABG, and FS. Investigation: DL, FAG, PGC, MAM, and ANP. Methodology: DL, MAM, ANP, ABG, IP, BC, FR, JDK, MJG, MS, and LB. Resources: AF, DM, CB, AL, MS, LB, J-ML, and LA. Writing original draft: DL, FAG, and PGC. Writing - review and editing: DL, MAM, ANP, ABG, FAG, and PGC

Funding This project was supported by Cancer Research UK (CRUK; C19212/ A16628, C19212/A911376), the CRUK Cambridge Centre (C9685/A25177), a Cambridge Commonwealth, European and International Trust PhD Scholarship, a European Institute of Innovation and Technology (EIT) health grant for Innovation for Personalized Cancer Medicine, the CRUK \& Engineering and Physical Sciences Research Council (EPSRC) Cancer Imaging Centre in Cambridge and Manchester (C197/A16465), Cancer Core Europe, and Addenbrooke's Charitable Trust. This research was also supported by the National Institute for Health Research Cambridge Biomedical Research Centre (RG85317). Special thanks to Professor Edwin Chilvers and Professor Klaus Okkenhaug for the PhD cosupervision of Doreen Lau; our research trial patients for supporting this study; Cambridge Cancer Trials Centre clinical trial coordinator David Bruce and Addenbrooke's Hospital Human Research Tissue Bank for assistance on FFPE samples; Stephanie Heasman, Sofia Koch, Christopher Bagnall, Hannah Hibbs, and Elizabeth Henley from AstraZeneca UK for the logistics support in pathology; and the Magnetic Resonance \& Spectroscopy Unit at Addenbrooke's Hospital for the technical support in MRI. We acknowledge the help of Gaspar Delso (GE Healthcare) and Dattesh Shanbhag (GE Global Research) for their MRI motion correction programming code and Dimitris Voukantsis for his advice on data visualization.

Competing interests No conflict of interest to declare with respect to the content of this work. AL, MS, LB, and J-ML are employees of AstraZeneca UK.

\section{Patient consent for publication Obtained.}

Ethics approval MelResist is a prospective study approved by the local institutional review board and research ethics committee (11/NE/0312) and managed within the Cambridge Clinical Trials Unit, Cambridge University Hospitals National Health Service Foundation Trust, Cambridge, UK. Written informed consent was obtained from all patients before enrolment.

Provenance and peer review Not commissioned; externally peer reviewed.

Data availability statement Data are available on reasonable request. Data may be obtained from a third party and are not publicly available. All data relevant to the study are included in the article or uploaded as supplementary information. Please contact the corresponding author Doreen Lau (la399@cam.ac.uk).

Supplemental material This content has been supplied by the author(s). It has not been vetted by BMJ Publishing Group Limited (BMJ) and may not have been peer-reviewed. Any opinions or recommendations discussed are solely those of the author(s) and are not endorsed by BMJ. BMJ disclaims all liability and responsibility arising from any reliance placed on the content. Where the content includes any translated material, BMJ does not warrant the accuracy and reliability of the translations (including but not limited to local regulations, clinical guidelines, terminology, drug names and drug dosages), and is not responsible for any error and/or omissions arising from translation and adaptation or otherwise.

Open access This is an open access article distributed in accordance with the Creative Commons Attribution 4.0 Unported (CC BY 4.0) license, which permits others to copy, redistribute, remix, transform and build upon this work for any purpose, provided the original work is properly cited, a link to the licence is given, and indication of whether changes were made. See https://creativecommons.org/ licenses/by/4.0/.

\section{ORCID iDs}

Doreen Lau http://orcid.org/0000-0002-7623-2401

Mary A McLean http://orcid.org/0000-0002-3752-0179

Andrew N Priest http://orcid.org/0000-0002-9771-4290

Andrew B Gill http://orcid.org//0000-0002-9287-9563
Jean-Martin Lapointe http://orcid.org/0000-0003-0141-4603

Pippa G Corrie http://orcid.org/0000-0003-4875-7021

Ferdia A Gallagher http://orcid.org/0000-0003-4784-5230

\section{REFERENCES}

1 Ribas A, Wolchok JD. Cancer immunotherapy using checkpoint blockade. Science 2018;359:1350-5.

2 Danson S, Hook J, Marshall H, et al. Are we over-treating with checkpoint inhibitors? Br J Cancer 2019;121:629-30.

3 Schachter J, Ribas A, Long GV, et al. Pembrolizumab versus ipilimumab for advanced melanoma: final overall survival results of a multicentre, randomised, open-label phase 3 study (KEYNOTE-006). Lancet 2017;390:1853-62.

4 Larkin J, Chiarion-Sileni V, Gonzalez R, et al. Five-year survival with combined nivolumab and ipilimumab in advanced melanoma. $N$ Engl J Med 2019;381:1535-46.

5 Larkin J, Chiarion-Sileni V, Gonzalez R, et al. Combined nivolumab and ipilimumab or monotherapy in untreated melanoma. $N$ Engl $J$ Med 2015;373:23-34.

6 Welsh SJ, Corrie PG. Biomarkers predicting for response and relapse with melanoma systemic therapy. Acta Derm Venereol 2020;100:adv00142.

7 Bedard PL, Hansen AR, Ratain MJ, et al. Tumour heterogeneity in the clinic. Nature 2013;501:355-64.

8 Gill AB, Rundo L, Wan JCM, et al. Correlating radiomic features of heterogeneity on CT with circulating tumor DNA in metastatic melanoma. Cancers 2020;12:3493.

9 Nishino M, Giobbie-Hurder A, Manos MP, et al. Immune-related tumor response dynamics in melanoma patients treated with pembrolizumab: identifying markers for clinical outcome and treatment decisions. Clin Cancer Res 2017;23:4671-9.

10 Wolchok JD, Hoos A, O'Day S, et al. Guidelines for the evaluation of immune therapy activity in solid tumors: immune-related response criteria. Clin Cancer Res 2009;15:7412-20.

11 Tan AC, Emmett L, Lo S, et al. FDG-pet response and outcome from antiPD-1 therapy in metastatic melanoma. Ann Oncol 2018;29:2115-20.

12 Chiou VL, Burotto M. Pseudoprogression and immune-related response in solid tumors. J Clin Oncol 2015;33:3541-3.

13 Pandit-Taskar N, Postow MA, Hellmann MD, et al. First-in-humans imaging with ${ }^{89} \mathrm{Zr}$-Df-IAB22M2C anti-CD8 Minibody in patients with solid malignancies: preliminary pharmacokinetics, biodistribution, and lesion targeting. J Nucl Med 2020;61:512-9.

14 Bensch F, van der Veen EL, Lub-de Hooge MN, et al. ${ }^{89} \mathrm{Zr}$ atezolizumab imaging as a non-invasive approach to assess clinical response to PD-L1 blockade in cancer. Nat Med 2018;24:1852-8.

15 Niemeijer AN, Leung D, Huisman MC, et al. Whole body PD-1 and PD-L1 positron emission tomography in patients with non-small-cell lung cancer. Nat Commun 2018:9:1-5.

16 Liu Z, Li Z, Qu J, et al. Radiomics of multiparametric MRI for pretreatment prediction of pathologic complete response to neoadjuvant chemotherapy in breast cancer: a multicenter study. Clin Cancer Res 2019;25:3538-47.

17 Bedair R, Priest AN, Patterson AJ, et al. Assessment of early treatment response to neoadjuvant chemotherapy in breast cancer using non-mono-exponential diffusion models: a feasibility study comparing the baseline and mid-treatment MRI examinations. Eur Radiol 2017;27:2726-36.

18 McRobbie DW, Moore EA, Graves MJ. Part II - The Specialist Stuff. In: Mri from picture to proton. 3rd edition. Cambridge University Press, 2017. ISBN: 9781107706958.

19 Hylton N. Dynamic contrast-enhanced magnetic resonance imaging as an imaging biomarker. J Clin Oncol 2006;24:3293-8.

20 Patterson DM, Zweifel M, Middleton MR, et al. Phase I clinical and pharmacokinetic evaluation of the vascular-disrupting agent OXi4503 in patients with advanced solid tumors. Clin Cancer Res 2012;18:1415-25.

21 Horsley L, Cummings J, Middleton M, et al. A phase 1 trial of intravenous 4-(N-(S-glutathionylacetyl)amino) phenylarsenoxide (GSAO) in patients with advanced solid tumours. Cancer Chemother Pharmacol 2013;72:1343-52.

22 Hu D-E, Beauregard DA, Bearchell MC, et al. Early detection of tumour immune-rejection using magnetic resonance imaging. $\mathrm{Br} \mathrm{J}$ Cancer 2003;88:1135-42.

23 Riegler J, Gill H, Ogasawara A, et al. VCAM-1 density and tumor perfusion predict T-cell infiltration and treatment response in preclinical models. Neoplasia 2019;21:1036-50.

24 Umemura Y, Wang D, Peck KK, et al. DCE-MRI perfusion predicts pseudoprogression in metastatic melanoma treated with immunotherapy. J Neurooncol 2020;146:339-46. 
25 Papaevangelou E, Almeida GS, Jamin Y, et al. Diffusion-weighted MRI for imaging cell death after cytotoxic or apoptosis-inducing therapy. Br J Cancer 2015;112:1471-9.

26 Barrett T, McLean M, Priest AN, et al. Diagnostic evaluation of magnetization transfer and diffusion kurtosis imaging for prostate cancer detection in a re-biopsy population. Eur Radiol 2018;28:3141-50.

27 Deen SS, Priest AN, McLean MA, et al. Diffusion kurtosis MRI as a predictive biomarker of response to neoadjuvant chemotherapy in high grade serous ovarian cancer. Sci Rep 2019;9:1-9.

28 Goodburn RJ, Barrett T, Patterson I, et al. Removing rician bias in diffusional kurtosis of the prostate using real-data reconstruction. Magn Reson Med 2020;83:2243-52.

29 Eisenhauer EA, Therasse P, Bogaerts J, et al. New response evaluation criteria in solid tumours: revised RECIST guideline (version 1.1). Eur J Cancer 2009;45:228-47.

30 Grzywa TM, Paskal W, Włodarski PK. Intratumor and intertumor heterogeneity in melanoma. Trans/ Oncol 2017;10:956-75.

31 Rabbie R, Ansari-Pour N, Cast O, et al. Multi-site clonality analysis uncovers pervasive heterogeneity across melanoma metastases. Nat Commun 2020;11:4306.
32 Ramón Y Cajal S, Sesé M, Capdevila C, et al. Clinical implications of intratumor heterogeneity: challenges and opportunities. $\mathrm{J} \mathrm{Mol} \mathrm{Med}$ 2020;98:161-77.

33 Queirolo P, Spagnolo F. Atypical responses in patients with advanced melanoma, lung cancer, renal-cell carcinoma and other solid tumors treated with anti-PD-1 drugs: a systematic review. Cancer Treat Rev 2017;59:71-8.

34 Wheen P, Armstrong R, Daly CA. Recent advances in T1 and T2 mapping in the assessment of fulminant myocarditis by cardiac magnetic resonance. Curr Cardiol Rep 2020;22:47.

35 Schiza A, Irenaeus S, Ortiz-Nieto F, et al. Evaluation of diffusionweighted MRI and FDG-PET/CT to assess response to AdCD40L treatment in metastatic melanoma patients. Sci Rep 2019;9:18069.

36 Reuben A, Spencer CN, Prieto PA, et al. Genomic and immune heterogeneity are associated with differential responses to therapy in melanoma. NPJ Genom Med 2017;2:10.

37 Klein D. The tumor vascular endothelium as decision maker in cancer therapy. Front Oncol 2018;8:367.

38 Gaustad J-V, Simonsen TG, Smistad R, et al. Early effects of low dose bevacizumab treatment assessed by magnetic resonance imaging. BMC Cancer 2015;15:900. 